\title{
Carvedilol among Pediatric Patients with Congestive Heart Failure
}

\author{
Butnariu Angela1 ${ }^{1}$, Vlase Laurian ${ }^{2}$, Leucuta Sorin ${ }^{2}$, Manuel Chira ${ }^{3}$ and Samaşca Gabriel ${ }^{4 *}$
}

${ }^{1}$ Department of Pediatrics III

${ }^{2}$ Department of Pharmaceutical Technology

${ }^{3}$ Heart Institute, Cluj-Napoca, Romania

${ }^{4}$ Department of Immunology; Iuliu Haţieganu University of Medicine and Pharmacy, Cluj-Napoca, Romani

Congestive heart failure $(\mathrm{CHF})$ as a result of systemic ventricular dysfunction represents the evolution of many heart disorders in childhood. Among these, dilated cardiomyopathy and some congenital cardiopathies are refractory to the known drug therapy, based on digitalic glycosides, diuretics and converting enzyme inhibitors. For these cases, the last therapeutic resource frequently remains heart transplantation. It is accepted that more than $50 \%$ of all children with CHF are candidates for transplantation [1]. However, transplantation involves many impediments, among which the lack of donors is the most important one. If beta-blocking agents were until not long ago contraindicated in patients with CHF, the studies carried out over the past 10 years show that beta-blocking agents improve left ventricular function, the clinical picture of $\mathrm{CHF}$ and survival in adults with CHF. A recent consensus on CHF in adults [2] currently recommends beta-blocking agents as part of the standard therapeutic protocol of $\mathrm{CHF}$. This remarkable turn from contraindications to consensus recommendations is due to the deeper understanding of the pathophysiology of heart failure.

The concept of adrenergic hyperactivity in congestive heart failure, the theoretical support of beta-blocking therapy

CHF is a symptomatic entity due to the incapacity of the heart to ensure the adequate perfusion and oxygenation of peripheral tissues. It manifests by fatigue, dyspnea, and signs of venous congestion. CHF is associated with severe ventricular dysfunction and pathological ventricular remodeling, changes that result in significant hemodynamic alterations. It is not surprising that doctors initially chose a treatment intended for these hemodynamic changes. Thus, traditional therapy of $\mathrm{CHF}$ recommends positive inotropic agents and peripheral vasodilators. But, the hemodynamic concept of the pathophysiology of heart failure proved to be incomplete. It was demonstrated that drugs with positive inotropic effects and phosphodiesterase inhibitors can result in the improvement of heart failure symptoms in the short term, but in the long term, they decrease survival $[3,4]$. At present, the concept of hemodynamic dysfunction has been associated with the concept of adrenergic hyperactivity. The activation of the sympathetic nervous system was described as one of the essential pathophysiological changes in patients with $\mathrm{CHF}$ or as one of the most important mechanisms responsible for heart failure progression [5]. In CHF, the activation of the adrenergic nervous system results in increased levels of circulating catecholamines [6,7], which accelerates the heart rate, increases blood pressure and myocardial contractility, effects that are positive in the short term. However, in time, adrenergic hyperactivity leads to the impairment of hemodynamics in the failing heart $[8,9]$. Direct detrimental effects include myocyte destruction and catecholamine induced necrosis $[10,11]$. The additional adrenergically mediated myocyte loss accelerates apoptosis, the programmed cell death process. The acceleration of the heart rate and the increase in myocardial contractility result in increased myocardial oxygen consumption, in increased wall stress and, with time, in the hypertrophy of the heart walls, with the alteration of the myocyte and the aggravation of heart failure. These physiological links are the theoretical support of the beneficial effects of $ß$-blocking agents that may lead to the prevention of chronic heart dysfunction in patients with CHF. Like many other classes of medications, beta blockers can be divided into three distinct groups. The first group consists of nonselective beta blockers without ancillary properties and includes such drugs as propranolol and timolol maleate. The second group consists of selective blockers of beta receptor subtypes without ancillary properties. This group includes metoprolol and atenolol. The third group consists of nonselective beta blockers that have the ancillary property of vasodilation. Included in this group are labetalol, carvedilol and bucindolol

Carvedilol: a non-selective beta-blocking agent, with antioxidant properties

Carvedilol is a nonselective beta-adrenoreceptor antagonist and

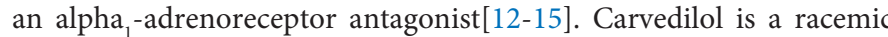
mixture with nonselective beta-adrenergic blocking activity produced by the S(-) enantiomer and alpha(1)-adrenergic blocking activity provided by both the $\mathrm{R}(+)$ and $\mathrm{S}(-)$ enantiomers in equal potency. It has no intrinsic sympathomimetic activity. Beta-adrenergic blocking agents are believed to improve cardiac failure by reducing circulating catecholamine concentrations. High levels of catecholamines, while useful in the short-term to increase cardiac output, result over time in increased cellular necrosis, increased myocardial oxygen consumption, ventricular hypertrophy, and fibrosis. By reducing catecholamines, beta-adrenergic blocking agents improve systolic function, decrease fibrotic remodeling of dilated ventricles, improve clinical symptoms, and have been associated with decreased hospitalization and mortality. It has been suggested that these agents also reduce QT dispersion, reducing the potential for sudden death from arrhythmia. Although documented in adults, this effect has not been demonstrated in children. Carvedilol provides the additional benefits of systemic arterial vasodilation through its alpha-blocking activity, anti-oxidant, and anti-endothelin effects.It has no intrinsic sympathomimetic activity. Bucindolol and carvedilol produce less "inverse agonism" than most other beta blockers. Inverse agonism is the ability of a beta blocker to inactivate active state receptors. The beta blockers with the most inverse agonism, like propranolol, produce the greatest negative chronotropic and inotropic effects. Thus, bucindolol and carvedilol produce relatively

*Corresponding author: Samaşca Gabriel, Department of Immunology; Iuliu Hațieganu University of Medicine and Pharmacy, Cluj-Napoca, Romania, E-mail: Gabriel.Samasca@umfcluj.ro

Received July 28, 2011; Accepted July 28, 2011; Published August 03, 2011

Citation: Angela B, Laurian V, Sorin L, Chira M, Gabriel S (2011) Carvedilol among Pediatric Patients with Congestive Heart Failure. Pharm Anal Acta 2:104e. doi:10.4172/2153-2435.1000104e

Copyright: (C) 2011 Angela B, et al. This is an open-access article distributed unde the terms of the Creative Commons Attribution License, which permits unrestricted use, distribution, and reproduction in any medium, provided the original author and source are credited. 
fewer negative chronotropic and inotropic effects when compared with beta blockers like propranolol. The beta-blocking actions of carvedilol are generally evident in humans within one hour of administration, and the alpha-mediated vasodilatory effects, manifested by decreased peripheral resistance and decreased blood pressure, are evident within about 30 minutes of administration. The clinical significance of alpha blockade in conjunction with beta blockade in the treatment of CHF is not known. The use of alpha blockers alone, however, does not appear to reduce mortality in patients with symptomatic CHF. At high dosages, carvedilol exerts calcium channel blocking activity. It also has significant antioxidant properties. Carvedilol inhibits the generation of oxygen free radicals and prevents low-density lipoprotein (LDL) oxidation, which, in turn, reduces the uptake of LDL into the coronary vasculature. This antioxidant activity may contribute to carvedilol's cardioprotective effects. In fact, compared with captopril, carvedilol has demonstrated similarly favorable effects on the lipid profiles of hypertensive patients with dyslipidemia [13-15]. In normal subjects and patients with hypertension, carvedilol is rapidly and completely absorbed after oral administration, achieving peak plasma concentrations within one to two hours. The volume of distribution is about 1.5 to 2 liters per kilogram of body weight, indicating substantial distribution into extravascular tissues. Absorption is delayed an additional 60 to 135 minutes when the drug is administered with food. Carvedilol undergoes extensive stereoselective first-pass hepatic metabolism. In plasma, 98 percent of the drug is bound to plasma proteins, predominantly to albumin. The terminal elimination half-life of carvedilol ranges from 7 to 10 hours in most subjects. Carvedilol is cleared by aromatic-ring oxidation and glucuronidation in the liver. The oxidative metabolites are then conjugated with glucuronide and sulfate,and the resulting conjugates are excreted in the bile and eliminated in the feces; only 16 percent is excreted in the urine. Some of the metabolites of carvedilol have beta-adrenoreceptor-antagonist activity, and one 4-hydroxyphenyl metabolite is approximately 13 times as potent as carvedilol in this regard. The metabolites also have weak vasodilator activity, but the clinical importance of this property is unknown. The metabolism of carvedilol is affected by genetic polymorphism of cytochrome P-450 2D6 activity, in that patients with low activity have higher plasma concentrations of $R$-carvedilol, a stereoisomer that has both alfa- and beta-adrenoreceptor-antagonist activity.Drugs that inhibit cytochrome P-450 2D6 activity, such as quinidine, paroxetine, fluoxetine, and propafenone, may also increase plasma carvedilol concentrations. Thus, patients taking these drugs may be at particularly high risk of hypotension due to excessive $\alpha$ -adrenoreceptor blockade. In contrast, plasma concentrations of $S$-carvedilol, which has only beta-adrenoreceptor-antagonist activity, are increased only slightly in patients with low cytochrome P-450 2D6 activity[13-15].Clearance of carvedilol is delayed in patients over 65 years of age. On average, their plasma carvedilol concentrations are 50 percent higher than in younger patients. Patients with liver disease also have high plasma carvedilol concentrations, but the half-life of the drug is unchanged. Patients with renal disease have slightly increased plasma carvedilol concentrations; the pharmacokinetics of carvedilol are not altered in patients undergoing hemodialysis. Drugs that increase cytochrome P-450 activity, such as rifampin, can accelerate the clearance of carvedilol. Hydrochlorothiazide does not influence the pharmacokinetics of carvedilol,and the pharmacokinetics of warfarin are not affected by carvedilol. Plasma digoxin concentrations rise slightly in patients given carvedilol. Patients with heart failure (particularly patients with New York Heart Association [NYHA] functional class IV heart failure) have higher plasma carvedilol concentrations than normal subjects of the same age, but the values overlap considerably between the two groups. The increase is higher for $S$-carvedilol than for $R$-carvedilol. Heart failure, by causing intestinal edema, may also reduce absorption of the drug. Since heart failure causes reduced cardiac output, depressed hepatic perfusion, and hepatic congestion, it could interfere with hepatic drug metabolism; these factors could influence the pharmacokinetics of carvedilol. However, in one study that assessed the pharmacokinetics of carvedilol in patients with heart failure, the stereoselective disposition of the drug was proportional to the dose over the therapeutic range (6.25 to $50 \mathrm{mg}$ per day).

\section{The use of carvedilol in pediatric patients}

In 2001, the first two articles documenting the use of carvedilol in children were published and others followed up [12,16-24]. Bruns et all reported the experiences of 6 centers who had treated a total of 46 children between 3 months and 19 years of age [16]. Thirty-seven of the patients had a dilated cardiomyopathy, while nine had congenital heart disease. Therapy was initiated at a dose of $0.08 \mathrm{mg} / \mathrm{kg}$ given twice daily, and titrated as needed to an average dose of $0.46 \mathrm{mg} / \mathrm{kg}$ $(0.92 \mathrm{mg} / \mathrm{kg} /$ day $)$. After three months of therapy, $67 \%$ of the patients had an improvement in their modified New York Heart Association (NYHA) class. Mean shortening fraction improved from $16.2 \%$ to $19 \%$. While the majority of the patients improved or remained stable, $30 \%$ were considered to have adverse outcomes (death or the need for transplantation or a ventricular-assist device) despite treatment. The authors concluded that carvedilol was a useful adjunct to standard therapy for pediatric cardiac failure. Gachara et al. published their experience with carvedilol in eight infants with dilated cardiomyopathy [17]. All patients were receiving digoxin, diuretics, and captopril. The average age at the start of therapy was $11+6.8$ months. An initial dose of $0.1 \mathrm{mg} / \mathrm{kg}$ was given twice daily. The dose was titrated every 24 hours as needed, with an average maintenance dose of $1.08+0.4 \mathrm{mg} / \mathrm{kg} /$ day. At follow-up $(4.5+2.2$ months $)$, the patients showed significant improvement over baseline, with an increase in left ventricular ejection fraction (LVEF) from $24.4+5 \%$ to $38.5+11 \%$. Five of the patients became asymptomatic, and two had only mild symptoms. In 2002, Azeka and colleagues conducted a prospective, randomized, double-blind, placebo-controlled trial of carvedilol in 22 children with refractory heart failure [18]. Carvedilol was initiated with a low dose of $0.01 \mathrm{mg} / \mathrm{kg} /$ day and increased as needed up to a target dose of $0.2 \mathrm{mg} /$ $\mathrm{kg} /$ day. At six months, there was a significant increase in LVEF in the carvedilol group (from $17.8 \%$ at baseline to $34.6 \%$ ). Modified NYHA class improved in 9 of the 14 patients, allowing them to be removed from the transplant waiting list. Patients in the placebo group showed no improvement; two died and two required transplantation. Laer et al. reported the results of an open-label trial of carvedilol in 15 patients (6 weeks to 19 years of age) with cardiac failure who failed traditional management [19]. Ten had dilated cardiomyopathy, and five had congenital heart disease. Carvedilol was started at $0.09 \mathrm{mg} / \mathrm{kg}$ twice daily and titrated at 2-week intervals to a maximum of adult dose of $50 \mathrm{mg} /$ day. The target maintenance dose was $0.7 \mathrm{mg} / \mathrm{kg} /$ day. Twelve patients completed the 6-month trial, with a significant improvement in LVEF from $36 \%$ to $54 \%$. Clinical symptom scores also showed statistically significant improvement. Mean arterial pressure declined from $75 \mathrm{~mm} \mathrm{Hg}$ to $60 \mathrm{~mm} \mathrm{Hg}$ and heart rate decreased from 116 beats/ min to 96 beats/min. During their open-label trial, Laer et al. compared the pharmacokinetic profile of carvedilol in the 15 children enrolled with nine healthy adults. In their study, the children exhibited higher maximum serum concentrations (average $16.9 \mathrm{ng} / \mathrm{ml}$ in children versus $11.2 \mathrm{ng} / \mathrm{ml}$ in adults), although AUC values were similar. Elimination 
half-life was significantly shorter in the pediatric patients than in the adults ( 2.9 versus 5.2 hours). When the pediatric patients were further divided into two groups (those $<3.5$ years and those older), the average elimination half-life was 2.2 hours in the younger children versus 3.6 hours in the older patients $(2,11)$. Williams et al. conducted a retrospective review of their experience with carvedilol and metoprolol in 12 children with left ventricular systolic dysfunction [20]. All patients had remained symptomatic on traditional therapy. Six patients were given metoprolol (0.4-2.4 mg/kg/day) and six received carvedilol (0.4$0.9 \mathrm{mg} / \mathrm{kg} /$ day). At six months, there was a significant increase in both shortening fraction (from $13+4 \%$ to $21+8 \%$ ) and LVEF (from $26+8 \%$ to $41+17 \%$ ). There were no significant differences between the patients given metoprolol and those given carvedilol. An early response to betaadrenergic blocker therapy was associated with increased survival and reduced need for transplantation. In 2003, Giardini et al. [21] studied the effects of carvedilol in nine children with dilated cardiomyopathy over a one-year period. Therapy was initiated at $0.05 \mathrm{mg} / \mathrm{kg} / \mathrm{day}$ and increased every 1-2 weeks to a target dose of $0.8 \mathrm{mg} / \mathrm{kg} /$ day. At 12-month follow-up, plasma concentrations of norephinephrine, dopamine, and aldosterone were significantly reduced compared to baseline. Positive effects on ventricular remodeling were noted, with significant reductions in end-diastolic and end-systolic diameters, and an increase in LVEF. Maunoury et al. demonstrated a similar benefit in 17 children (average age $39 \pm 57$ months) given carvedilol [22]. Treatment was initiated with a dose of $0.01 \mathrm{mg} / \mathrm{kg} /$ day and titrated to $0.2 \mathrm{mg} / \mathrm{kg} /$ day. After 6 months, LVEF increased from 26 $+11 \%$ to $43+17 \%$, with 13 of the patients exhibiting more than a $10 \%$ improvement. In addition, the study showed a $38 \%$ increase in cardiac uptake of iodine-123 metaiodobenzylguanidine, representing a significant improvement in cardiac adrenergic neuronal function. In 2003, Ratnapalan and colleagues studied the interaction in eight children.Digoxin clearance declined from $153.0+92.3 \mathrm{ml} / \mathrm{min} / 1.73 \mathrm{~m}^{2}$ to $80.6+23.9 \mathrm{ml} / \mathrm{min} / 1.73 \mathrm{~m}^{2}$ after four days of concomitant carvedilol administration [23]. All patients had an increase in serum digoxin concentrations, with two patients having levels above the therapeutic range. Based on their observations, the authors recommend reducing the dose of digoxin by $25 \%$ in children receiving carvedilol. Rusconi et al. reported their experience over a 3-year period [24]. Carvedilol was added to standard therapy in patients with a persistent LVEF $\leq 40 \%$. The review included 24 children between 1 day and 16.5 years of age. The average initial and final doses were $0.15+0.09 \mathrm{mg} / \mathrm{kg} /$ day and $0.98+0.26 \mathrm{mg} / \mathrm{kg} /$ day, respectively. Two patients discontinued therapy, one for asthma and the other for worsening cardiac failure. In the remaining patients, there was a significant increase in LVEF from $24.6+7.6 \%$ to $42.2+14.2 \%$. Fifteen patients $(68 \%)$ had improvement in their NYHA functional class. One patient died and 3 received transplants. In addition to these reports, a multicenter trial of carvedilol in children with heart failure is currently underway (Shaddy). With an expected enrollment of 150 children, this placebo-controlled study has been designed to address a composite of clinical outcomes including mortality, hospitalization, and symptomatic improvement, as well as measuring indices of ventricular function [1].

The integrated aspects of pharmacokinetics, metabolic drug interactions and clinical efficacy of carvedilol therapy in infants and children are important aspects for a better understanding and management of the pharmacotherapy in pediatric population. By the way it is important to elucidate other aspects of these essential steps in order to optimise the therapy in children.

\section{References}

1. Shaddy RE, Curtin EL, Sower B, Tani LY, Burr J, et al. (2002) The Pediatric Randomized Carvedilol Trial in Children with Heart Failure: rationale and design. Am Heart J Sep 144: 383-389.

2. Packer M, Cohn JN (1999) Consensus recommendations for the management of chronic heart failure. Am J Cardiol 83: 39A- 42A.

3. Packer M (1996) Beta-blockade in the management of chronic heart failure Eur Heart J 17: 21-23.

4. Pfeffer MA, Stevenson LW (1996) Beta-adrenergic blockers and survival in heart failure [Editorial]. N Engl J Med 334: 1396-1397.

5. Colucci WS, PackerM, Bristow MR, Gilbert EM, Cohn JN, et al. (1996) Carvedilol inhibits clinical progression in patients with mild symptoms of heart failure. Circulation 94:2800-2806.

6. Chidsey CA, Harrison DC, Braunwald E (1996) Augmentation of the plasma norepinephrine response to exercise in patients with congestive heart failure. $\mathrm{N}$ Engl J Med 267: $650-654$

7. Francis GS, Benedict C, Johnstone DE, Kirlin PC, Nicklas J, et al. (1990) Comparison of neuroendocrine activation in patients with left ventricular disfunction with and without congestive heart failure. Circulation 82: 1724-1729.

8. Cohn JN, Levine TB, Olivari MT, Garberg V, Lura D, et al. (1984) Plasma norepinephrine as a guide to prognosis in patients with chronic congestive heart failure. N Engl J Med 311: $819-823$.

9. Sharma M, Nair MNG, Jatana SK, Shani BN (2003) Congestive heart failure in infants and children. MJAFI 59: 228-233.

10. Yates JC, Beamish RE, Dhalla NS (1981) Ventricular dysfunction and necrosis produced by adrenochrome metabolite of epinephrine: relation to pathogenesis of cathecolamine cardiomiopathy. Am Heart J 102: 210 - 221.

11. Mann DL, Kent RL, Parsons B, Cooper G (1992) Adrenergic effects on the biology of the adult mammalian cardiocyte. Circulation 85: 790- 804

12. Buck ML (2005) Use of carvedilol in children with cardiac failure. Pediatric Pharmacotherapy 11: 1-7.

13. Vanderhoff BT, Ruppel HM, Amsterdam PB (1998) Carvedilol: the new role of beta blockers in congestive heart failure. Amer Fam Physician 58: 1-12.

14. Ruffolo RR, Gellai M, Heible JP, Willette RN, Nichols AJ (1990) The pharmacology of carvedilol. Eur J Clin Pharmacol 38: 82-88.

15. Frishman WH (1998) Carvedilol. Drug Therapy 339: 1759-1765

16. Bruns LA, Chrisant MK, Lamour JM (2001) Carvedilol as therapy in pediatric heart failure: an initial multicenter experience. J Pediatr 138: 505-511.

17. Gachara N, Prabhakaran S, Srinivas S (2001) Efficacy and safety of carvedilo in infants with dilated cardiomyopathy: a preliminary report. Indian Heart J 53 : 74-78.

18. Azeka E, Ramires JAF, Valler C (2002) listing of infants and children from the heart transplantation waiting list after carvedilol treatment. J Am Coll Cardiol 40: 2034-2038

19. Laer S, Mir TS, Behn F (2002) Carvedilol therapy in pediatric patients with congestive heart failure: a study investigating clinical and pharmacokinetic parameters. Am Heart J 143: 916-922

20. Williams RV, Tani LY, Shaddy RE (2002) Intermediate effects of treatment with metoprolol or carvedilol in children with left ventricular systolic dysfunction. $J$ Heart Lung Transplant 21: 906-909.

21. Giardini A, Formigari R, Bronzetti G (2003) Modulation of neurohormona activity after treatment of children in heart failure with carvedilol. Cardiol Young 13: $333-336$

22. Maunoury C, Acar P, Sidi D (2003) Use of ${ }^{123}$ I-MIBG scintigraphy to assess the impact of carvedilol on cardiac adrenergic neuronal function in childhood dilated cardiomyopathy. Eur J Nucle Med Mold Imaging 30: 1651-1656.

23. Ratnapalan S, Griffiths K, Costei AM (2003) Digoxin carvedilol interactions in children. J Pediatr 142: 572-574.

24. Rusconi P, Gomez-Marin O, Rossique-Gonzalez M (2004) Carvedilol in children with cardiomyopathy: 3-year experience at a single institution. J Heart Lung Transplant 23: 832-838. 